\title{
HUBBLE SPACE TELESCOPE OBSERVATIONS OF BAADE'S WINDOW
}

\author{
W. A. BAUM, Univ. of Washington, Seattle, WA 98195, USA \\ R. M. LIGHT, Univ. of California, Santa Cruz, CA 95064, USA
}

J. HOLTZMAN, D. HUNTER, T. KREIDL, Lowell Obs., Flagstaff, AZ 86001, USA

E. J. O'NEIL, JR., Kitt Peak Nat. Obs., Tucson, AZ 85726, USA

E. J. GROTH, Princeton Univ., Princeton, NJ 08544, USA

This is a status report on a continuing program using the Hubble Space Telescope (HST) Wide-Field Camera (WFC) to probe the stellar population of the Galactic bulge to fainter magnitudes. We seek the mean age of the stars and the initial mass function (IMF). Galactic bulge stars offer the only opportunity to investigate the IMF of a super metal-rich population. They are 100 times closer than the next nearest sample.

On 18 August 1991, two long HST-WFC exposures $\left(1600^{s}\right.$ and $\left.2000^{s}\right)$ were made with the F555W filter and two $\left(2000^{s}\right.$ each) with the F785LP filter. The WFC is the f/12.9 mode of the "WF/PC" (Westphal 1982; MacKenty 1992), and it covers $2.5 \times 2.5$ arcmin at an image scale of $0.10^{\prime \prime}$ per pixel. Our WFC field is located at $18^{h} 03^{m} 10^{s}$, $-29^{\circ} 51^{\prime} 43^{\prime \prime}(2000)$. It was selected to lie in the apparently most transparent part of Baade's Window, 11.4' NNW from the imbedded globular star cluster NGC 6522, and $3.8^{\circ}$ from the direction to the Galactic center. We should expect $A_{V}$ for the HST-WFC field to be less than the average for Baade's Window.

After standard WF/PC reduction procedures (Lauer 1989) were applied to the images, the F555W set and the F785LP set were each stacked (co-added), and DAOPHOT was used for stellar photometry. Instrumental magnitudes in the F555W and F785LP passbands were transformed to the Johnson-Cousins $V, I$ system using WF/PC calibration data published by Harris et al. (1991).

Our color-magnitude diagram in Figure 1 is based on profile-fitting photometry. This CM diagram is limited at the bright end by the onset of CCD saturation at $V \sim 18$ mag. At the faint end, it ceases to be meaningful below $V \sim 23.5 \mathrm{mag}$, where photometric errors are rising steeply. Terndrup's (1988) CM diagram, which bottomed at $V \sim 20$ mag, can be merged with the top of ours, and they join well if we assume Terndrup's $V-I$ to include a few hundredths of a magnitude more reddening than ours. His field was located $3.1^{\prime} \mathrm{NW}$ of NGC 6522 , or about $8^{\prime}$ south of our HST field.

Contamination by the foreground disk population was discussed by Terndrup. In our case, it would average only about 10 stars per $0.25-\mathrm{mag}$ bin over the range of our data. That is of no importance below $V \sim 20 \mathrm{mag}$ in Figure 1, but it is a significant contributor around 18th and 19th magnitude, and it masks the bulge turnoff.

By slicing our CM diagram into $\Delta V=0.25-\mathrm{mag}$ bins and finding the median value of $V-I$ in each bin, we obtain the ridge-line plotted in Figure 2. Theoretical isochrones, such as those of Green, Demarque, and King (1987) fit the main sequence (i.e., the $20.0<V<23.5 \mathrm{mag}$ portion of this ridge-line) impressively well, but the fit is sensitive to any zero-point error in the magnitude scales and to the validity of the models. Moreover, the mean age of the population is not uniquely determined by the fit, because for each choice of mean age, one can find associated values of $A_{V}$ and $R_{0}$ that permit an acceptable fit. Each choice must thus be judged by the plausibility of those associated values. Taking abundances $Z=0.04$ and $Y=0.30$, we find, for example, 
that a $10 \mathrm{Gyr}$ isochrone fits if $A_{V} \approx 1.2 \mathrm{mag}$ and $R_{0} \approx 7.4 \mathrm{kpc}$. By Buonanno's (1986) criterion, the turnoff in this case should fall at $V_{t} \approx 19.8$. For each $+1 \mathrm{Gyr}$ in assumed mean age, the corresponding differentials are $\Delta A_{V} \sim-0.02 \mathrm{mag}, \Delta R_{0} \sim-0.06 \mathrm{kpc}$, and $\Delta V_{t} \sim+0.08 \mathrm{mag}$. Although the present results appear to favor a scenario in which star formation in the bulge continued longer than in the halo, the uncertainties are large, and a 15 Gyr mean age cannot be ruled out.

To produce a $V$ luminosity function, star counts were made in $0.25-\mathrm{mag}$ bins, and a very large number of simulated stars with appropriate PSFs were inserted, one at a time, to derive completeness corrections as a function of magnitude. (The simulated stars also served to check against systematic errors in the photometry.) Preliminary analysis yields a corrected luminosity function for the main sequence in Baade's Window which does not differ much from that in the solar neighborhood.

\section{References}

Buonanno, R. 1986. Mem.Soc.Astron.Ital. 57, 333.

Green, E. M., Demarque, P., and King, C. R. 1987. The Revised Yale Isochrones and Luminosity Functions (New Haven: Yale Univ. Observatory).

MacKenty, J. W. 1992. HST Wide-Field/Planetary Camera Instrument Handbook (Baltimore: Space Telescope Science Institute).

Harris, H. C., Baum, W. A., Hunter, D. A., and Kreidl, T. J. 1991. Astron.J. 101, 677. Lauer, T. R. 1989. Publ.Astron.Soc.Pac. 101, 445.

Terndrup, D. M. 1988. Astron.J. 96, 884.

Westphal, J. A. 1982. In The Space Telescope Observatory NASA CP-2244, ed. D. N. B. Hall (Washington: NASA Scientific and Technical Information Branch), 28.

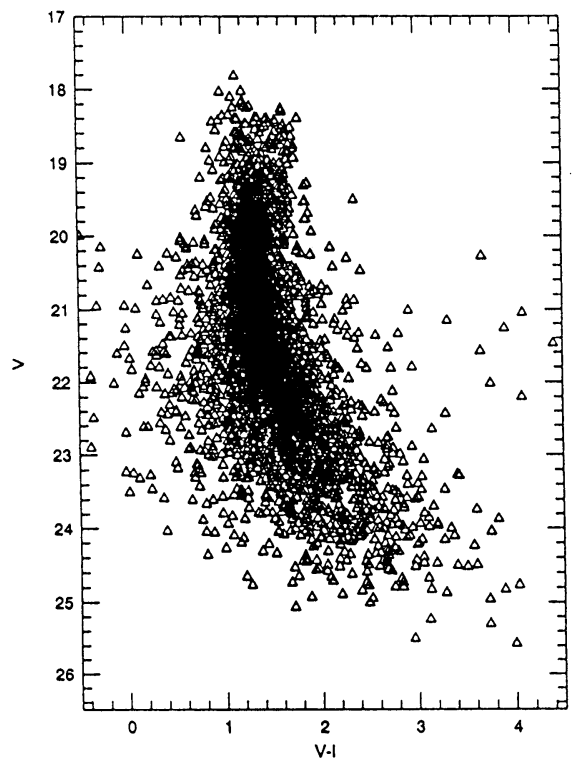

Fig 1. CM diagram of Baade's Window.

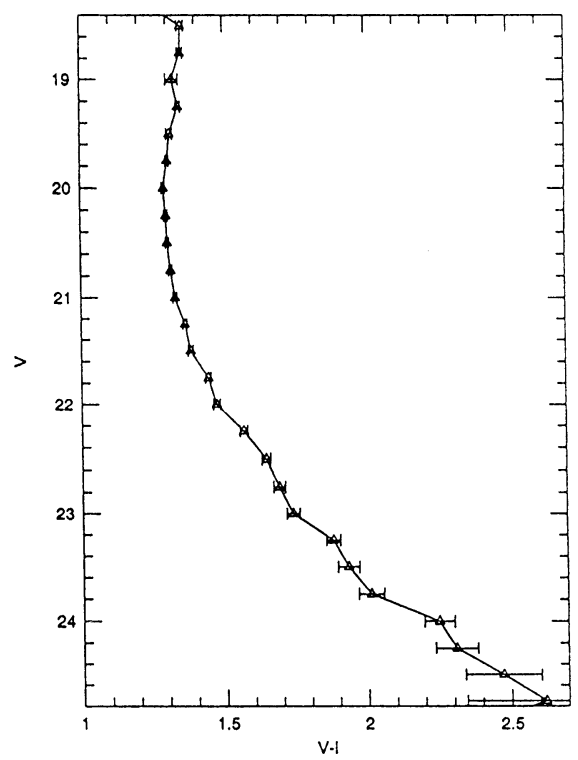

Fig 2. Ridge-line of the CM diagram. 